\title{
Neumonía por Mycoplasma pneumoniae complicada con anemia hemolítica por aglutininas frías
}

\section{Mycoplasma pneumoniae pneumonia complicated by cold agglutinin hemolytic anemia}

\author{
Marcos Arango, Mario Arenas, Octavio Martínez • Bogotá, D.C.
}

\section{Resumen}

Paciente mujer de 36 años de edad, quien consulta por síntomas respiratorios de dos semanas de evolución, con diagnóstico de ingreso de neumonía adquirida en la comunidad. Evolución clínica complicada con anemia hemolítica por hemaglutininas frías que hace sospechar infección neumónica por Mycoplasma pneumoniae, confirmada mediante serología. Tras curso corto de prednisona oral, ácido fólico y antibioticoterapia, se presenta resolución de la anemia y mejoría clínica de la neumonía. Este informe de caso resalta la elevada amplitud térmica de las aglutininas frías como condición para generar hemólisis inmune y la ausencia de reticulocitosis compensatoria de la hemólisis, complicaciones inusuales en neumonía por Mycoplasma pneumoniae, pero determinantes de la severidad de la anemia. Además, enfatiza la utilidad de los títulos de aglutininas frías en el diagnóstico de neumonía adquirida en la comunidad debida a Mycoplasma pneumoniae. (Acta Med Colomb 2013; 38: 91-94)

Palabras clave: crioaglutininas, hemólisis, Mycoplasma pneumoniae, neumonía.

\begin{abstract}
We present the case of a 36 years old female patient, who consults for respiratory symptoms during two weeks, with admission diagnosis of community-acquired pneumonia. The clinical course was complicated by hemolytic anemia by cold hemagglutinin that suggests pneumonic infection by Mycoplasma pneumoniae, confirmed by serology. After short course of oral prednisone, folic acid and antibiotic therapy, resolution of anemia and clinical improvement of the pneumonia were obtained. This case report highlights the high temperature range of cold agglutinins as a condition for generating immune hemolysis and the absence of compensatory reticulocytosis of hemolysis, which are rare pneumonia complications of Mycoplasma pneumoniae, but that are determinants of the severity of the anemia. It also emphasizes the usefulness of cold agglutinin titers in the diagnosis of community-acquired pneumonia due to Mycoplasma pneumoniae. (Acta Med Colomb 2013; 38: 91-94).
\end{abstract}

Key words: cold agglutinins, hemolysis, Mycoplasma pneumoniae, pneumonia.
Dres. Marcos Arango Barrientos y Mario Arenas Mantilla: Residentes de Hematología Departamento de Medicina Interna, Facultad de Medicina, Universidad Nacional de Colombia; Dr. Octavio Martínez Betancur: Profesor Asociado Departamento de Medicina Interna, Facultad de Medicina ,Universidad Nacional de Colombia. Bogotá, D.C. Correspondencia. Dr. Dr. Octavio Martínez E-mail: omartinezb@unal.edu.co

Recibido: 16/VI/2012 Aceptado: 25/II/2013

\section{Introducción}

La neumonía por Mycoplasma pneumoniae usualmente tiene un curso clínico febril agudo, autolimitado, asociado con tos no productiva, y raramente evoluciona con complicaciones clínicas que requieran manejo hospitalario. Entre las manifestaciones extrapulmonares, la más frecuente es la hematológica, específicamente anemia hemolítica asociada con aglutininas frías (1-3).
La hemólisis que ocurre tras la neumonía causada por Mycoplasma pneumoniae, es causada por la activación del complemento por aglutininas frías policlonales del tipo inmunoglobulina $\mathrm{M}(\operatorname{IgM})$ con especificidad contra el antígeno $I$ de la membrana del eritrocito, el cual se torna autoinmunogénico al ligarse con el Mycoplasma pneumoniae (4-6).

Las manifestaciones clínicas varían grandemente entre los pacientes, dependiendo de la amplitud térmica de las 
aglutininas frías. En general, la patogenicidad de la aglutinina fría es más dependiente de la amplitud térmica que de la concentración del anticuerpo en suero. Si la amplitud térmica excede $29^{\circ}-30^{\circ} \mathrm{C}$, los eritrocitos se aglutinan en la circulación de las partes acrales del cuerpo aun a temperatura ambiente, y aparece hemólisis por activación del complemento mediada por IgM. Una proporción de glóbulos rojos recubiertos de $\mathrm{C} 3 \mathrm{~b}$, es destruida por las células del sistema fagocítico mononuclear, principalmente en el hígado y, en la superficie de los eritrocitos supervivientes, el C3b es clivado, dando lugar a gran número de moléculas $\mathrm{C} 3 \mathrm{~d}$ sobre la superficie celular (7-11).

Estos mecanismos explican por qué la prueba de Coombs directa es fuertemente positiva para $\mathrm{C} 3 \mathrm{~d}$ en pacientes con hemólisis mediada por aglutininas frías y, en la mayoría, negativa para $\operatorname{IgM}$ e $\operatorname{IgG}(8)$. La hemólisis por aglutininas frías puede diagnosticarse si están presentes; i) evidencia clínica de anemia hemolítica adquirida; ii) prueba de Coombs directa positiva con $\mathrm{C} 3$; iii) prueba de Coombs directa negativa con IgG; iv) presencia de una aglutinina fría con reactividad de al menos $30^{\circ} \mathrm{C}$ en salina o albúmina; y v) título de aglutinina fría $\left(\mathrm{a} 4^{\circ} \mathrm{C}\right) \geq 256$ (7).

Se presenta una paciente con diagnóstico de ingreso de neumonía adquirida en la comunidad cuya evolución clínica complicada con anemia hemolítica por hemaglutininas frías hace sospechar infección neumónica por Mycoplasma pneumoniae, confirmada mediante serología. Este informe de caso resalta la elevada amplitud térmica de las aglutininas frías como condición para generar hemólisis inmune y la ausencia de reticulocitosis compensatoria de la hemólisis,

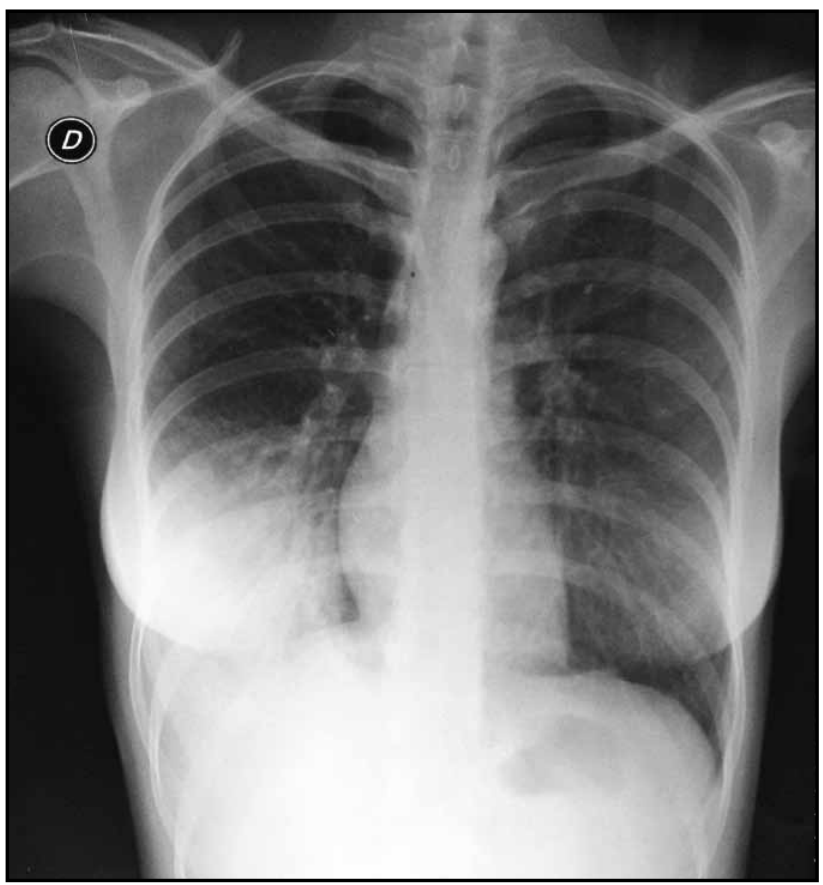

Figura 1. Opacidad en base pulmonar derecha de patrón alveolar. Radiografía posteroanterior de tórax. Neumonía por Mycoplasma pneumoniae complicada con hemólisis por aglutininas frías. complicaciones inusuales en neumonía por Mycoplasma pneumoniae, pero determinantes de la severidad de la anemia. Además, enfatiza la utilidad de los títulos de aglutininas frías en el diagnóstico de neumonía adquirida en la comunidad debida a Mycoplasma pneumoniae.

\section{Caso clínico}

Mujer de 36 años de edad, procedente de Bogotá, quien labora como encuadernadora de libros. Consulta en el servicio de urgencias del Hospital San Carlos (Bogotá) por un cuadro clínico de dos semanas de evolución, consistente en astenia y malestar general, asociados a fiebre cuantificada hasta de $39^{\circ} \mathrm{C}$ y tos productiva de material hialino en escasa cantidad. Tres días antes del ingreso su estado general se deterioró, con intenso malestar y postración, asociados a dolor lumbar y aparición de orina oscura. Como antecedente médico, informa psoriasis con sólo manejo tópico con corticosteroides. Ninguna medicación en el curso de la enfermedad. Los signos vitales al ingreso fueron: frecuencia cardiaca 90 latidos por minuto, frecuencia respiratoria 20 respiraciones por minuto, presión arterial $90 / 60 \mathrm{mmHg}$ y temperatura de $37^{\circ} \mathrm{C}$. Al examen físico inicial destacaba la palidez de mucosas, la ausencia de adenomegalias periféricas, la presencia de hallazgos sugestivos de consolidación pulmonar en la base derecha, la ausencia de hepatomegalia y esplenomegalia, y la presencia de lesiones cutáneas en extremidades y torso compatibles con su antecedente conocido de psoriasis. La radiografía del tórax (Figura 1), confirmó la ocupación alveolar sospechada clínicamente. Además, se documentó la presencia de una anemia macrocítica arregenerativa (índice de reticulocitos corregido $<2 \%$ ), con trombocitosis y leucocitosis asociadas (Tabla 1). Se inició manejo antibiótico con ampicilina/sulbactam y claritromicina.

Tabla 1. Evolución temporal de los resultados de laboratorio. Paciente con diagnóstico de neumonía por Mycoplasma pneumoniae complicada con hemólisis por aglutininas frías.

\begin{tabular}{|c|c|c|c|c|c|}
\hline Paraclínicos & Día 1 & Día 4 & Día 5 & Día 10 & Día 31 \\
\hline Hemoglobina (g/dL) & 7.1 & 6.9 & 6.2 & 7.9 & 14.1 \\
\hline Hematócrito (\%) & 21 & 20 & 15 & 23 & 42.1 \\
\hline $\mathrm{VCM}^{*}(\mathrm{fL})$ & 102 & 89 & 100 & 95 & 90 \\
\hline $\operatorname{IPRC} * *(\%)$ & 0.6 & & 4 & 8.4 & 1.8 \\
\hline Leucocitos (x 109/L) & 13.7 & 13.4 & 10.3 & 13.0 & 8.2 \\
\hline Neutrófilos (x 10\%/L) & 12.5 & 10.4 & 7.1 & 9.1 & 4.5 \\
\hline Linfocitos (x 10\%/L) & 0.8 & 2.0 & 2.5 & 3.1 & 3.3 \\
\hline Plaquetas (x 109/L) & 529 & 652 & 729 & 600 & 321 \\
\hline Creatinina (mg/dL) & 0.7 & & & & \\
\hline Bilirrubina total $(\mathrm{mg} / \mathrm{dL})$ & 2.7 & & & 0.8 & \\
\hline Bilirrubina directa $(\mathrm{mg} / \mathrm{dL})$ & 0.8 & & & 0.1 & \\
\hline DHL*** (UI/L) & 606 & & & 343 & \\
\hline \multicolumn{6}{|c|}{$\begin{array}{l}\text { *VCM. Volumen corpuscular medio. } \\
\text { **IPRC. Índice porcentual de reticulocitos corregidos. } \\
\text { ***DHL. Deshidrogenasa láctica. }\end{array}$} \\
\hline
\end{tabular}


Tras el inicio de la terapia con antibióticos la paciente informa mejoría clínica, pero la cifra de hemoglobina desciende $1 \mathrm{~g} / \mathrm{dL}$ al quinto día de hospitalización, respecto al valor de ingreso. La hiperbilirrubinemia indirecta, el aumento de deshidrogenasa láctica y la disminución de niveles de haptoglobina sérica $(3.7 \mathrm{mg} / \mathrm{dL}$ - Normal 30 200), hacen sospechar hemólisis autoinmune asociada a posible infección pulmonar por Mycoplasma pneumoniae. La prueba de Coombs directa fue positiva, y la prueba de Coombs directa fraccionada fue positiva para C3d (3+), negativa para $\mathrm{C} 3 \mathrm{c}$, negativa para $\mathrm{IgG}$ y positiva débil para IgM. Los títulos de crioaglutininas a $4^{\circ} \mathrm{C}$ fueron de $1: 1.024$, y los anticuerpos IgM contra Mycoplasma pneumoniae (ELISA) fueron positivos. Resultados negativos de anticuerpos antinucleares, anticuerpos anticardiolipinas, anticoagulante lúpico y factor reumatoide.

La paciente recibió suplencia con ácido fólico vía oral y prednisona $0.5 \mathrm{mg} / \mathrm{kg} /$ día, obteniéndose mejoría progresiva de la concentración de hemoglobina y de la reticulocitopenia de ingreso y, tras completar esquema antibiótico, fue dada de alta. Evaluada tres semanas después en consulta ambulatoria, se documenta corrección completa de la anemia y ausencia de manifestaciones pulmonares, y se suspende prednisona.

\section{Discusión}

Entre las formas secundarias de anemia hemolítica causada por aglutininas frías, la neumonía por Mycoplasma pneumoniae es la más frecuente. Aunque se desarrolla neumonía clínica en sólo 3-10\% de las personas infectadas por Mycoplasma pneumoniae, 15\% del total de neumonías en la población general, son debidas a este germen (8).

De 33\%-76\% de los pacientes con infección por Mycoplasma pneumoniae tienen títulos séricos de aglutininas frías mayores que 1:64 (a $4^{\circ} \mathrm{C}$ ) o los desarrollan durante la convalecencia. Puesto que $83 \%$ de los pacientes con infección por Mycoplasma pneumoniae tienen positividad de la prueba de Coombs directa y $64 \%$ cursan con reticulocitosis $(>2 \%)$, se sugiere que la hemólisis subclínica es común, con una frecuencia de anemia hemolítica manifiesta (disminución de hemoglobina $\geq 2 \mathrm{~g} / \mathrm{dL}$ ) que fluctúa entre 1.4 y $17 \%$, asociada con un marcado compromiso pulmonar (12-15).

La prueba de Coombs directa es positiva para C3d y, usualmente, los anticuerpos IgM no se detectan in vitro a $37^{\circ} \mathrm{C}$ puesto que se disocian de los eritrocitos tras la unión del C3, excepto cuando la amplitud térmica de la IgM es elevada, en cuyo caso la hemólisis es de mayor severidad y se sucede predominantemente en el compartimiento intravascular $(7,14,16)$. La positividad de la prueba de Coombs directa para IgM en el caso presentado, habla de una elevada amplitud térmica de la aglutinina, lo que explica la presencia de anemia clínicamente manifiesta y sugiere la aparición de orina oscura por hemoglobinuria.

Entre los análisis de laboratorio indicadores de hemólisis se incluyen una reducción de haptoglobina sérica (marcador de aumento de destrucción eritrocitaria) y un incremento del recuento reticulocitrario (signo de aumento reactivo de la eritropoyesis). Los niveles séricos de haptoglobina, al ser una proteína de fase aguda, pueden estar normales o elevados a pesar de la hemólisis, consecuencia de la patología inflamatoria. De otra parte, la haptoglobina sérica puede estar baja en ausencia de hemólisis, por ejemplo en casos de enfermedad hepática crónica o de hemólisis intramedular. Así, el diagnóstico de hemólisis se debe realizar en un contexto clínico sindromático, en el cual la haptoglobina sérica no siempre es de utilidad (17). Una particularidad del presente caso fue la falta de respuesta medular apropiada observada al inicio del cuadro, evidenciada por un bajo índice reticulocitario. La reticulocitosis no siempre está presente al momento del diagnóstico de anemia hemolítica, y hasta un $25 \%$ de pacientes cursan con recuentos normales o bajos. La principal razón es el retardo de la médula ósea para compensar la hemólisis, una deficiencia concomitante de ácido fólico, o una eritroblastopenia por anticuerpos dirigidos contra progenitores eritropoyéticos (17). En el contexto de hemólisis por aglutininas frías se ha descrito específicamente eritropoyesis inefectiva inmunológicamente mediada en ausencia de maduración megaloblástica y displasia medular (18).

El tratamiento con esteroides en la hemólisis por aglutininas frías asociada a neumonía por Mycoplasma pneumoniae está controvertido, particularmente por tratarse de una complicación usualmente no severa y autolimitada con el tratamiento antibiótico para la infección. El beneficio de los esteroides parece notarse en pacientes con aglutininas frías IgM a bajo título o con elevada amplitud térmica (11).

Títulos elevados de aglutininas frías en pacientes con neumonía atípica adquirida en la comunidad sugieren Mycoplasma pneumoniae. Es un aforismo clínico que "a mayor título de aglutininas frías por encima de 1:64 en un paciente con neumonía adquirida en la comunidad (NAC), es más probable que las aglutininas frías sean debidas a Mycoplasma pneumoniae". Para optimizar la detección de aglutininas frías en pacientes con NAC por Mycoplasma pneumoniae, se deben realizar durante la primera y segunda semanas mediciones interdiarias de títulos de aglutininas frías, puesto que tales títulos se dan tempranamente en el curso de la NAC por Mycoplasma pneumoniae. Un paciente con NAC con un título de aglutininas frías mayor de 1:128 es altamente indicativo de Mycoplasma pneumoniae, si se excluyen otras causas no infecciosas de altos títulos de aglutininas frías. Por supuesto, la ausencia de títulos elevados de aglutininas frías no excluye la NAC por Mycoplasma pneumoniae (19).

\section{Referencias}

1. Linz DH, Tolle SW, Elliot DL. Mycoplasma pneumoniae pneumonia. West J Med 1984; 140: 895 - 900.

2. Lind K. Manifestations and complications of Mycoplasma pneumoniae disease: A review. Yale J Biol Med 1983; 56: 461 - 468.

3. Tsuruta R, Kawamura Y, Inoue T, Kasaoka S, Sadamitsu D, Maekawa T. 
Corticosteroid therapy for hemolytic anemia and respiratory failure due to $M y$ coplasma pneumoniae pneumonia. Intern Med 2002; 41: 229 - 232.

4. McNicholl FP. Clinical syndromes associated with cold agglutinins. Transf Science 2000; 22: 125 - 133 .

5. Sokol RJ, Booker DJ, Stamps R, Walewska R. Cold haemagglutinin disease: clinical significance of serum haemolysins. Clin Lab Haem 2000; 22: 337 - 344.

6. Petz LD, Garratty G. Classification and clinical characteristics of autoimmune hemolytic anemias. En: Immune hemolytic anemias. $2^{\text {nd }}$ ed. Philadelphia: Churchill Livingstone; 2004. p. $61-131$.

7. Petz LD. Cold antibody autoimmune hemolytic anemias. Blood Rev 2008; 22: $1-15$.

8. Berentsen S, Tjonnfjord GE. Diagnosis and treatment of cold agglutinin mediated autoimmune hemolytic anemia. Blood Rev 2012; 26: 107 - 115.

9. Rosse WF, Adams JP. The variability of hemolysis in the cold agglutinin syndrome. Blood 1980; 56: 409 - 416.

10. Gertz MA. Management of cold haemolytic syndrome. Br J Haematol 2007; 138: $422-429$.

11. Gerhs BC, Friedberg RC. Autoimmnue hemolytic anemia. Am J Hematol 2002; 69: $258-271$.

12. Chu CS, Braun SR, Yarbo JW, Hayden MR. Corticosteroid treatment of hemo- lytic anemia associated with Mycoplasma pneumoniae pneumonia. South Med J 1990; 83: $1106-1108$

13. Ali NJ, Sillis M, Andrews BE, Jenkins PF, Harrison BD. The clinical spectrum and diagnosis of Mycoplasma pneumoniae infection. Quart J Med 1986; 58: 241251.

14. Kottayam R, Rozenberg G, Cohn RJ. Unusual haematologic manifestations of Mycoplasma pneumoniae infection. J Paed Child Health 2007; 43: 80- 82.

15. Daxböck F, Zedtwitz-Liebenstein K, Brugmann H, Graninger W. Severe hemolytic anemia and excessive leukocytosis masking mycoplasma pneumonia. Ann Hematol 2001; 80: 180 - 182.

16. Khan FY, Ayassin M. Mycoplasma pneuominae associated with severe autoimmune hemolytic anemia: case report and literature review. Braz J Infect Disease 2009; 13: 77 - 79 .

17. Valent P, Lechner K. Diagnosis and treatment of autoimmune haemolyticanaemias in adults: a clinical review. Wien Klin Wochenschr 2008; 120: 136 - 151.

18. Cazzola M, Barosi G, Ascari E. Cold haemagglutinin disease with severe anaemia, reticulocytopenia and erithroid bone marrow. Scand J Haematol 1983; 30: $25-29$

19. Cunha BA. The clinical diagnosis of Mycoplasma pneumonia: the diagnostic importance of highly elevated serum cold agglutinins. Eur J Clin Microbiol Infect Dis 2008; 27: 1017 - 1019 . 